\title{
Original Research \\ UV-4B potently inhibits replication of multiple SARS-CoV-2 strains in clinically relevant human cell lines
}

\author{
Evelyn J. Franco ${ }^{1,2}$, Kelly L. Warfield ${ }^{3}$, Ashley N. Brown ${ }^{1,2, *}$ \\ ${ }^{1}$ Department of Medicine, Institute for Therapeutic Innovation, College of Medicine, University of Florida, Orlando, FL 32827, USA \\ ${ }^{2}$ Department of Pharmaceutics, College of Pharmacy, University of Florida, Orlando, FL 32827, USA \\ ${ }^{3}$ Emergent BioSolutions, Gaithersburg, MD 20879, USA \\ *Correspondence: Ashley.Brown@medicine.ufl.edu (Ashley N. Brown) \\ Academic Editor: Sang Heui Seo \\ Submitted: 26 October 2021 Revised: 10 December 2021 Accepted: 17 December 2021 Published: 5 January 2022
}

\begin{abstract}
Background: SARS-CoV-2 is the coronavirus responsible for the COVID-19 pandemic. Although it poses a substantial public health threat, antiviral regimens against SARS-CoV-2 remain scarce. Here, we evaluated the antiviral potential of UV-4B, a host targeting antiviral, against SARS-CoV-2 in clinically relevant human cell lines. Methods: Cells derived from human lung (A549 cells transfected with human angiotensin converting enzyme 2 receptor (ACE2; ACE2-A549)) and colon (Caco-2) were infected with either a wild type or beta variant strain of SARS-CoV-2 and exposed to various concentrations of UV-4B. Supernatant was sampled daily and viral burden was quantified by plaque assay on Vero E6 cells. Results: Therapeutically feasible concentrations of UV-4B inhibited the replication of

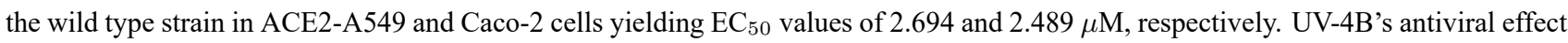
was also robust against the beta variant in both cell lines (ACE2-A549 $\mathrm{EC}_{50}: 4.369 \mu \mathrm{M}$; Caco-2 $\mathrm{EC}_{50}: 6.816 \mu \mathrm{M}$ ). Conclusions: These results highlight UV-4B's antiviral potential against several strains of SARS-CoV-2.
\end{abstract}

Keywords: UV-4B; Antiviral; Host targeting; SARS-CoV-2; COVID-19; Variant

\section{Introduction}

Severe Acute Respiratory Syndrome Coronavirus 2 (SARS-CoV-2) is the novel coronavirus responsible for the COVID-19 pandemic [1]. Since its emergence in late 2019, SARS-CoV-2 has spread to every continent, causing more than 150 million cases and over 3 million deaths as of April 2021 [2]. Most infected patients will experience mild to moderate disease however, risk for severe infection increases in older patients and those with comorbid conditions [3]. Infected patients commonly exhibit symptoms including fever, muscle pain, headache, cough, shortness of breath, and gastrointestinal (GI) distress. Severe cases of infection may lead to pneumonia, acute respiratory distress syndrome (ARDS), and respiratory failure [1,3].

Throughout the course of the pandemic, several variant strains of SARS-CoV-2 have emerged. The circulation of variants of concern include those from the United Kingdom, South Africa, Brazil, and India. The variants contain mutations in the spike protein that not only increase viral transmissibility, but also potentially render these strains less susceptible to the protective effect elicited by approved vaccines and monoclonal antibody therapies [4,5], highlighting the urgent need for antiviral regimens with activity against multiple strains of SARS-CoV-2.

In spite of SARS-CoV-2's emergence as a major threat to global public health, antiviral therapies approved to treat infection remain scarce. Currently, mild to moderate cases of infection are managed through supportive care mea- sures and, only one antiviral, the RNA polymerase inhibitor remdesivir, has received FDA approval for the treatment of severe cases of COVID-19 infection requiring hospitalization $[6,7]$.

UV-4B, the hydrochloride salt form of the iminosugar UV-4 (N-(9-methoxynonyl)-1-deoxynojirimycin; MONDNJ), is a broad-spectrum antiviral that has demonstrated virustatic or virucidal activity against several RNA viruses including dengue virus and influenza virus [8-10]. UV4B is a host targeting antiviral that disrupts the viral replication cycle through competitive inhibition of host cell endoplasmic reticulum $\alpha$-glucosidase I and II enzymes. This inhibitory effect is thought to prevent proper folding and processing of glycoproteins, ultimately leading to decreased viral assembly/release or changes in viral infectivity [8]. Since formation of replication competent SARS$\mathrm{CoV}-2$ virions is dependent on the production of glycoproteins, the antiviral UV-4B was selected as a promising antiviral candidate for these studies.

Here, the antiviral activity of UV-4B was evaluated against a wild type strain (USA-WA1/2020) and the beta variant strain of SARS-CoV-2 in two human cell lines. Angiotensin converting enzyme 2 (ACE2) receptor expressing A549 cells (ACE2-A549) are a human lung cell line that were selected since SARS-CoV-2 predominantly targets the lung and other tissues of the respiratory tract [11]. Caco-2 (human colorectal adenocarcinoma) cells, which naturally express the ACE2 receptor, were employed because SARS- 
CoV-2 infection is known to cause gastrointestinal distress and because of evidence supporting viral replication in the GI tract [11-13].

The ultimate goal of this study is to demonstrate the antiviral activity of UV-4B against wild type and beta variant strains of SARS-CoV-2 in two human cell lines that serve as in vitro models of tissues targeted by SARS-CoV-2 in man [11-13], providing a potential therapeutic option for the treatment of COVID-19.

\section{Materials and methods}

\subsection{Cells, virus and antivirals}

A549 cells stably expressing the angiotensin converting enzyme 2 (ACE2) receptor (ACE2-A549 cells) [14] were a kind gift from Dr. Shinji Makino. ACE2-A549 cells were maintained in Dulbecco's modified Eagle medium (DMEM) (Hyclone; Logan, UT, USA) supplemented with $10 \%$ fetal bovine serum (FBS) (Sigma Aldrich; St. Louis, MO, USA) and 1\% penicillin-streptomycin solution (Hyclone; Logan, UT, USA). Caco-2 cells (ATCC HTB-37) were purchased from the American Type Culture Collection (ATCC; Manassas, VA, USA) and cultured in minimum essential medium (MEM) (Corning Cellgro; Manassas, VA, USA) supplemented with 20\% FBS (Sigma Aldrich; St. Louis, MO, USA) and 1\% penicillin-streptomycin solution (Hyclone; Logan, UT, USA). Finally, Vero E6 cells (ATCC CRL-1586) were obtained from the American Type Culture Collection (ATCC; Manassas, VA, USA) and maintained in MEM (Corning Cellgro; Manassas, VA, USA) supplemented with 10\% FBS (Sigma Aldrich; St. Louis, MO, USA) and $1 \%$ penicillin-streptomycin solution (Hyclone; Logan, UT, USA). Cells were incubated at $37^{\circ} \mathrm{C}, 5 \% \mathrm{CO}_{2}$, and subcultured twice weekly to maintain subconfluency.

The wild type SARS-CoV-2 strain, isolate USAWA1/2020, and beta variant, isolate hCoV-19/South Africa/KRISP-EC-K005321/2020 were obtained from Biodefense and Emerging Infectious Research Resources Repository (BEI Resources; Manassas, VA, USA). Viral stocks were propagated on Vero E6 cells as previously described [15].

UV-4B was kindly provided by Emergent BioSolutions (Gaithersburg, MD, USA). UV-4B stocks of 2809.8 $\mu \mathrm{M}(1000 \mu \mathrm{g} / \mathrm{mL})$ were prepared in sterile deionized water.

\subsection{Antiviral evaluations}

ACE2-A549 or Caco-2 cells were plated onto six-well plates at a cell density of $1 \times 10^{6}$ cells per well. Cells were infected with either the wild type or the beta variant strain of SARS-CoV-2 at varying multiplicities of infection (MOIs) to account for cell line dependent differences in permissiveness to infection. For assays conducted with the wild type strain, an MOI of 0.03 was employed for ACE2-A549 cells, and Caco-2 cells were infected at an MOI of 0.006. ACE2A549 and Caco-2 cells were infected with the beta vari- ant strain of SARS-CoV-2 at MOIs of 0.0075 and 0.006 , respectively. Virus was allowed to adsorb onto cells for one hour, then the viral inoculum was removed and monolayers were washed twice with warm PBS to remove unbound virus. $3 \mathrm{~mL}$ of drug containing medium at UV-4B concentrations ranging from $0-400 \mu \mathrm{M}$ was added to each well. Plates were maintained at $37{ }^{\circ} \mathrm{C}$ and $5 \% \mathrm{CO}_{2}$. Viral supernatant was sampled daily for four days, clarified by high speed centrifugation, and frozen at $-80^{\circ} \mathrm{C}$ until the end of the study. Infectious viral burden was quantified by plaque assay on Vero E6 cells as previously described [15]. Briefly, Vero E6 cells were seeded onto six-well plates and allowed to attach overnight. The following day, viral supernatants were serially diluted tenfold in MEM and 100 microliters of each dilution was inoculated onto confluent cell monolayers. Plates were incubated for 1 hour at $37^{\circ} \mathrm{C}$ and $5 \% \mathrm{CO}_{2}$, then a primary overlay consisting of MEM, $5 \% \mathrm{FBS}$, and $0.5 \%$ agar was added to each well. Two days after infection, a secondary overlay containing MEM, 1\% FBS, $0.5 \%$ agar, and $0.007 \%$ neutral red was added to each well. Plaques were counted the following day and infectious viral burden was expressed as plaque forming units per milliliter $(\mathrm{PFU} / \mathrm{mL})$.

\subsection{Cell viability assays}

UV-4B cytotoxicity was measured with the commercially available WST-1 cell proliferation assay (Roche Diagnostics GmbH; Mannheim, Germany). ACE2-A549 or Caco- 2 cells were seeded onto 96-well plates at concentrations of 5000, and 15,000 cells/well, respectively and incubated overnight at $37{ }^{\circ} \mathrm{C}, 5 \% \mathrm{CO}_{2}$. The following day, medium in each well was removed and $200 \mu \mathrm{L}$ of UV$4 \mathrm{~B}$ containing medium at concentrations ranging from 0 $400 \mu \mathrm{M}$ was added to each well. Cells were incubated in the presence of drug for three days, then WST-1 reagent was added to each well as per manufacturer recommendations. Absorbance was detected 2-4 hours after addition of reagent using a SpectraMax M5 microplate reader (Molecular Devices; San Jose, CA, USA).

\subsection{Statistical analysis}

$\mathrm{EC}_{50}$ values were determined by fitting an inhibitory sigmoid $\mathrm{E}_{\max }$ model to the area under the viral burden-time curve $\left(\mathrm{AUC}_{V B}\right)$ over the entire time course of the experiment using GraphPad Prism software version 7.02 (GraphPad Software; La Jolla, CA, USA). $\mathrm{CC}_{50}$ values were calculated by graphing absorbance values at 72 hours against drug concentration and fitting an inhibitory sigmoid $\mathrm{E}_{\max }$ model to the data using GraphPad Prism software. Cell viability is reported as percent cell viability relative to an untreated control. An unpaired student's $t$-test with a Welch's correction was performed using GraphPad Prism software (version 7.02, GraphPad Software, La Jolla, CA, USA) comparing the mean of each treatment group to that of the control. A $p$ value of less than 0.05 was considered significant. 


\section{Results}

\subsection{UV-4B is not toxic to ACE2-A549 and Caco-2 cells at evaluated concentrations}

Cell proliferation assays were conducted in both cell lines to evaluate UV-4B for potential cytotoxicity. Our results demonstrated UV-4B was not toxic to ACE2-A549 cells $\left(\mathrm{CC}_{50}>400 \mu \mathrm{M}\right)$ (Table 1 ) since percent cell viability remained comparable to the uninfected control at all studied concentrations (Fig. 1A). UV-4B concentrations ranging from 1.56 to $100 \mu \mathrm{M}$ were similarly nontoxic to Caco-2 cells (Fig. 1B) but $400 \mu \mathrm{M}$ caused slight declines in cell viability (mean value of $100 \%$ with a $95 \%$ confidence interval (C.I.) of $81.94-118.1 \%$ for the no-treatment control versus $82.86 \%$ with a $95 \%$ C.I. of $77.32-88.41 \%$ for the $400 \mu \mathrm{M}$ therapy arm), although these differences between the no-treatment control arm and the $400 \mu \mathrm{M}$ experimental arm were not statistically significant $(p=0.059)$. These data may indicate that high concentrations of UV-4B produce a cytostatic effect in this cell line $\left(\mathrm{CC}_{50}>400 \mu \mathrm{M}\right)$ (Table 1).
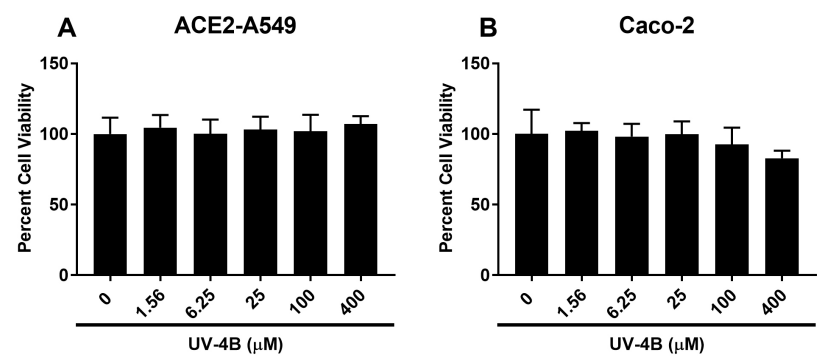

Fig. 1. Effect of UV-4B on cell viability of ACE2-A549 and Caco-2 cells. ACE2-A549 (A) and Caco-2 (B) cells were treated with different concentrations of UV-4B. Cell viability was measured after three days using the commercially available WST-1 assay according to manufacturer recommendations. Cell viability is reported as percent cell viability relative to an untreated control. Columns represent the mean of 6 independent samples, error bars represent one standard deviation.

\section{$3.2 U V-4 B$ suppressed replication of a wild type strain of $S A R S-C o V-2$}

UV-4B effectively inhibited replication of the wild type SARS-CoV-2 strain in ACE2-A549 cells, yielding an $\mathrm{EC}_{50}$ value of $2.69 \mu \mathrm{M}$ (Table 1). In the absence of treatment, viral titers reached a peak of $6.44 \log _{10} \mathrm{PFU} / \mathrm{mL}$ on day 4 (Fig. 2A). The lowest concentration of UV-4B evaluated $(1.56 \mu \mathrm{M})$ caused modest declines in infectious viral burden of $0.7 \log _{10}$ PFU/mL. Antiviral effectiveness increased dramatically following exposure to higher drug concentrations. For example, 6.25 $\mu \mathrm{M}$ UV-4B caused a nearly 1000 -fold decrease in peak titers relative to the control. UV-4B concentrations $\geq 25 \mu \mathrm{M}$ further curbed production of infectious virus, as viral titers were maintained at or below the assay limit of detection throughout the 4day course of therapy. UV-4B's antiviral effect was similarly robust against the wild type strain in Caco-2 cells since drug treatment yielded considerable, sustained viral inhibition at all evaluated concentrations (Fig. 2B). On day 4, when peak titers are achieved in this cell line, a $1.4 \log _{10}$ $\mathrm{PFU} / \mathrm{mL}$ decrease in infectious viral burden was observed following treatment with $1.56 \mu \mathrm{M}$ UV-4B. Similar to results in ACE2-A549 cells, infectious titers remained at or below the assay limit of detection at UV-4B concentrations $\geq 25$ $\mu \mathrm{M}$. UV-4B achieved an $\mathrm{EC}_{50}$ value equivalent to $2.49 \mu \mathrm{M}$ in this cell line (Table 1).

\subsection{The beta variant strain of SARS-CoV-2 is also sensitive to $U V-4 B$ effect}

ACE2-A549 and Caco-2 cells infected with the beta variant strain of SARS-CoV-2 were also sensitive to UV4B's antiviral effect but the degree of inhibition elicited by drug treatment varied slightly between both strains. In ACE2-A549 cells, UV-4B exhibited an $\mathrm{EC}_{50}$ value of 4.37 $\mu \mathrm{M}$ (Table 1). Drug concentrations of $1.56 \mu \mathrm{M}$ delayed but did not completely inhibit viral replication since antiviral effect was lost by day 3, when peak viral titers were achieved in this cell line. Antiviral activity was more substantial at drug concentrations $\geq 6.25 \mu \mathrm{M}$. For example, $6.25 \mu \mathrm{M}$ UV-4B decreased peak titers by approximately 2.4 $\log _{10}$ PFU/mL, while the highest concentrations of UV-4B evaluated (100 and $400 \mu \mathrm{M})$ completely arrested the production of infectious virus in this cell line (Fig. 2C). In Caco- 2 cells, the beta variant achieved peak titers of 4.94 $\log _{10} \mathrm{PFU} / \mathrm{mL}$ in the absence of treatment. In contrast to results against the wild type strain, drug concentrations $\geq 6.25$ $\mu \mathrm{M}$ were required to successfully inhibit replication of the beta variant since infectious titers following treatment with $1.56 \mu \mathrm{M} U \mathrm{UV}-4 \mathrm{~B}$ were identical to the no-treatment control arm (Fig. 2D). On day 4, effective UV-4B regimens (6.25$400 \mu \mathrm{M})$ decreased titers by 0.97 and $1.87 \log _{10} \mathrm{PFU} / \mathrm{mL}$ at 6.25 and $400 \mu \mathrm{M}$, respectively. The $\mathrm{EC}_{50}$ value in this cell line was $6.82 \mu \mathrm{M}$ (Table 1$)$.

\section{Discussion}

The emergence of SARS-CoV-2 in late 2019 initiated a global health crisis that has affected millions [1]. The appearance of viral variants with the potential to escape the protective effect afforded by currently available vaccines and monoclonal antibody therapies highlights the urgent need for antiviral regimens that exhibit activity against multiple strains of SARS-CoV-2. In this work, the $\alpha$ glucosidase inhibitor, UV-4B, was evaluated for its antiviral potential against two strains of SARS-CoV-2, a wild-type strain and the beta strain that harbors mutations in the viral spike protein. Because this virus is known to infect multiple organ systems [11], studies were carried out in two cell lines representative of human target tissues. 
Table 1. Inhibitory sigmoid $\mathbf{E}_{\max }$ model parameter estimates, including fifty percent effective concentration ( $\left(\mathrm{EC}_{50}\right)$, and fifty percent cytotoxic concentration $\left(\mathrm{CC}_{50}\right)$ of $\mathrm{UV}-4 \mathrm{~B}$ in $\mathrm{ACE} 2-\mathrm{A549}$ and Caco-2 cells.

\begin{tabular}{|c|c|c|c|c|c|c|}
\hline & \multirow[b]{2}{*}{$R^{2 a}$} & \multicolumn{3}{|c|}{ Inhibitory Sigmoid $\mathrm{E}_{\max }$ Model Parameters } & \multirow[b]{2}{*}{$\mathrm{EC}_{50}(\mu \mathrm{M})$} & \multirow[b]{2}{*}{$\mathrm{CC}_{50}(\mu \mathrm{M})$} \\
\hline & & $\mathrm{E}_{\text {con }}{ }^{b}$ & $\mathrm{E}_{\max }{ }^{c}$ & Hill & & \\
\hline ACE2-A549 Cells & & & & & & $>400$ \\
\hline Wild-Type & 0.999 & $14.860(0.14)^{d}$ & $6.865(0.09)$ & $1.709(0.12)$ & $2.694(0.14)$ & \\
\hline Beta Variant & 0.999 & $19.020(0.23)$ & $6.643(0.17)$ & $1.464(0.11)$ & $4.369(0.27)$ & \\
\hline Caco-2 Cells & & & & & & $>400$ \\
\hline Wild-Type & 0.971 & $13.08(0.66)$ & $6.819(0.66)$ & $0.866(0.45)$ & $2.489(1.32)$ & \\
\hline Beta Variant & 0.997 & $12.81(0.19)$ & $7.686(0.16)$ & $1.907(0.44)$ & $6.816(0.70)$ & \\
\hline
\end{tabular}

\footnotetext{
${ }^{a} R^{2}$ represents the goodness of fit of the model to the data.

${ }^{b} \mathrm{E}_{c o n}$ represents the effect in the absence of drug, or the control effect. The units for $\mathrm{E}_{c o n}$ are $\log _{10^{-}}$ plaque forming units per $\mathrm{mL}(\mathrm{PFU} / \mathrm{mL}) \cdot$ days.

${ }^{c} \mathrm{E}_{\max }$ represents the maximum effect, or greatest reduction in viral output, resulting from drug expo-

sure. The units for $\mathrm{E}_{\max }$ are $\log _{10}$ - plaque forming units per $\mathrm{mL}(\mathrm{PFU} / \mathrm{mL}) \cdot$ days.

${ }^{d}$ The value in parenthesis corresponds to the estimated standard error of the model.
}

UV-4B inhibited replication of a wild type and the beta variant strain of SARS-CoV-2 in ACE2-A549 and Caco-2 cells at low micromolar concentrations in the absence of cytotoxicity. Further investigation of UV-4B's anti-SARS$\mathrm{CoV}-2$ activity is warranted for several reasons. First, because potent activity against both viral strains was achieved at therapeutically feasible concentrations. A phase I trial evaluation of oral UV-4B safety, tolerability, and pharmacokinetics (NCT02061358) showed a one-time dose of $1000 \mathrm{mg}$ was associated with a $\mathrm{C}_{\text {ave }}$ of $5.58 \mu \mathrm{M}$ and a $\mathrm{C}_{\max }$ of $40 \mu \mathrm{M}$ in plasma [16]. The binding of UV-4B to human plasma proteins is approximately $50 \%$ (personal communication, Dr. Tony Treston, Emergent BioSolutions, Inc.). Thus, free drug $\mathrm{C}_{\max }$ concentrations of $20 \mu \mathrm{M}$ still greatly exceed those required to elicit a substantial antiviral effect. Second, because these results are in agreement with previous studies which show that UV-4B has antiviral activity against SARS-CoV-2 in other cell types, including Vero E6 cells, and that different $\alpha$-glucosidase inhibitors are effective at inhibiting SARS-CoV-2 in infected ACE2A549 cells [17,18]. The broad spectrum activity exhibited by UV-4B and related $\alpha$-glucosidase inhibitors hinges on the reliance of many enveloped viruses on the host cell glycosylation machinery for production of glycoproteins [19]. SARS-CoV-2 is similarly dependent on host cell glycoprotein processing because the spike protein, which is crucial for viral entry into cells, is heavily glycosylated [17,20]. In a study conducted by Clarke et al. [17], levels of SARS-CoV-2 spike protein (USA-WA1/2020) and viral genome copies were reduced following treatment with celgosivir, another $\alpha$-glucosidase inhibitor, in Vero E6 cells. It is hypothesized that UV-4B acts through a similar mechanism and drug induced misfolding of the spike protein hinders virion assembly/release. Modification of viral infectivity may also contribute to observed antiviral effect, since work done with SARS-CoV suggests treatment with $\alpha$-glucosidase inhibitors modulates viral infectivity by producing viral particles that express less spike protein [21].

Although therapeutically feasible concentrations of UV-4B inhibited replication of a wild type and variant strain of SARS-CoV-2, the degree of viral inhibition varied between strains in both ACE2-A549 and Caco-2 cell lines since the beta variant was less sensitive to UV-4B effect at the lowest concentrations evaluated. Spike protein mutations present in the beta strain associated with greater viral transmissibility may contribute to between strain variability in UV-4B susceptibility. For example, the D614G mutation may enhance viral infectivity and increase viral entry by increasing spike protein density of released viral particles [22]. The D614G mutation is also predicted to enhance viral virulence by increasing glycosylation at the nearby 616 residue $[23,24]$. This variant also expresses several mutations (N501Y, E484K, and K417N substitutions) in the receptor binding domain that are associated with enhanced binding affinity to the ACE2 receptor [4,5]. It is plausible that alpha-glucosidase enzymes are only partially inhibited at low UV-4B concentrations and the mutations in the variant strain that enhance viral infectivity or increase binding affinity to the ACE2 receptor nullify drug effect at these concentrations.

A potential limitation to this study was all UV-4B regimens were evaluated at static drug concentrations, this does not accurately represent the pharmacokinetic profiles achieved following drug administration in man. Future studies using the hollow fiber infection model will allow for the simulation of pharmacokinetic (PK) profiles associated with oral UV-4B administration in man and provide insight into this drug's anti-SARS-CoV-2 activity under dynamic concentrations. 


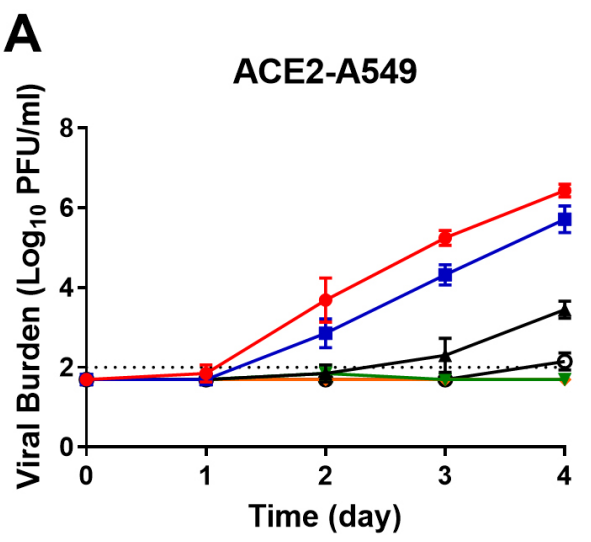

\section{Wild Type}
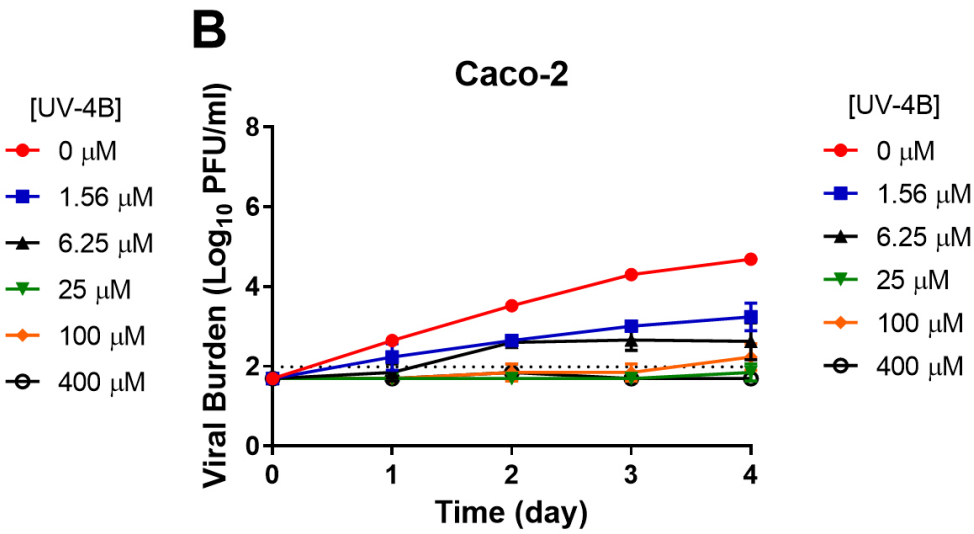

\section{Beta Variant}
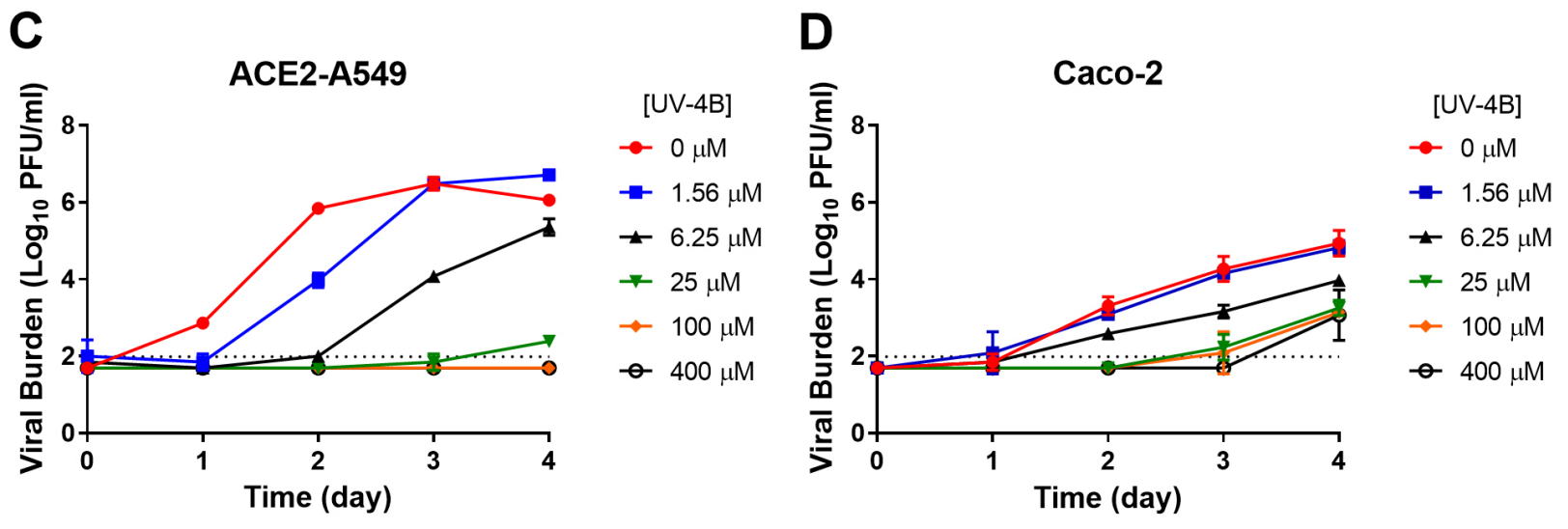

Fig. 2. Antiviral activity of UV-4B against SARS-CoV-2 in ACE2-A549, and Caco2 cells. ACE2-A549 (A) and Caco-2 (B) cells were infected with the wild type strain of SARS-CoV-2 at multiplicities of infection (MOIs) of 0.03, and 0.006, respectively. ACE2-A549 (C) and Caco-2 (D) cells were infected with the beta variant strain of SARS-CoV-2 at MOIs of 0.0075 and 0.006, respectively. Infected cells were treated with different concentrations of UV-4B at concentrations ranging from 0-400 $\mu \mathrm{M}$. Infectious viral burden was quantified by plaque assay on Vero E6 cells, and reported as $\log _{10}$ plaque forming units per milliliter $(\mathrm{PFU} / \mathrm{mL})$. Data points represent the mean of two independent samples and error bars correspond to one standard deviation. The dashed line signifies the assay limit of detection.

\section{Conclusions}

The results of this study demonstrate that UV-4B substantially inhibited the replication of both a wild type and a variant strain of SARS-CoV-2 at therapeutically feasible concentrations. Future studies will focus on evaluating UV-4B for its effectiveness against other mutant strains of SARS-CoV-2 as monotherapy as well as in combination with other anti-SARS-CoV-2 agents.

\section{Abbreviations}

SARS-CoV-2, Severe Acute Respiratory Syndrome Coronavirus 2; COVID-19, coronavirus disease 2019; ARDS, acute respiratory distress syndrome; ACE2, angiotensin converting enzyme 2; ACE2-A549, ACE2 receptor expressing A549 cells; DMEM, Dulbecco's modified
Eagle medium; FBS, fetal bovine serum; GI, Gastrointestinal; MEM, minimum essential medium; MOI, multiplicity of infection; PBS, phosphate buffered saline; PK, pharmacokinetic; PFU/mL, plaque forming units per milliliter; $\mathrm{EC}_{50}$, fifty percent effective concentration; $\mathrm{CC}_{50}$, fifty percent cytotoxic concentration.

\section{Author contributions}

Conceptualization, ANB; methodology, EJF and ANB; formal analysis, EJF and ANB; investigation, EJF; resources, ANB and KLW; writing-original draft, EJF and ANB; writing-review and editing, EJF, KLW and ANB; supervision, ANB. 


\section{Ethics approval and consent to participate}

Not applicable.

\section{Acknowledgment}

The authors would like to thank Anthony Treston from Emergent BioSolutions for reviewing the manuscript. The following reagent was obtained through BEI Resources, NIAID, NIH: SARS-Related Coronavirus 2, isolate hCoV-19/South Africa/KRISP-EC-K005321/2020, NR-54008, contributed by Alex Sigal and Tulio de Oliveira. The following reagent was deposited by the Centers for Disease Control and Prevention and obtained through BEI Resources, NIAID, NIH: SARS-Related Coronavirus 2, isolate USA-WA1/2020, NR-52281.

\section{Funding}

This research was funded by the Institute for Therapeutic Innovation, University of Florida.

\section{Conflict of interest}

The authors declare no conflict of interest. KLW is employed by Emergent BioSolutions.

\section{References}

[1] Rehman MFU, Fariha C, Anwar A, Shahzad N, Ahmad M, Mukhtar S, et al. Novel coronavirus disease (COVID-19) pandemic: a recent mini review. Computational and Structural Biotechnology Journal. 2021; 19: 612-623.

[2] World Health Organization. WHO Coronavirus (COVID-19) Dashboard. 2021. Available at: https://covid19.who.int/ (Accessed: 5 May 2021)

[3] Centers for Disease Control and Prevention. Symptoms of COVID-19. Centers for Disease Control and Prevention. 2021. Available at: https://www.cdc.gov/coronavirus/2019-ncov/sy mptoms-testing/symptoms.html (Accessed: 5 May 2021).

[4] Tegally H, Wilkinson E, Giovanetti M, Iranzadeh A, Fonseca V, Giandhari J, et al. Detection of a SARS-CoV-2 variant of concern in South Africa. Nature. 2021; 592: 438-443.

[5] Zhou D, Dejnirattisai W, Supasa P, Liu C, Mentzer AJ, Ginn $\mathrm{HM}$, et al. Evidence of escape of SARS-CoV-2 variant B.1.351 from natural and vaccine-induced sera. Cell. 2021; 184: 23482361.e6.

[6] Centers for Disease Control and Prevention. Information for Clinicians on Investigational Therapeutics for Patients with COVID-19. Centers for Disease Control and Prevention. 2020. Available at: https://www.cdc.gov/coronavirus/2019-ncov/hcp/ therapeutic-options.html (Accessed: 9 May 2021).

[7] US Food \& Drug Administration. Frequently Asked Questions for Veklury (remdesivir). 2020. Available at: $\quad$ https://www.fda.gov/media/137574/download\#: : text=On $\% 20$ October $\% 2022 \% 2 \mathrm{C} \% 202020 \% 2 \mathrm{C} \% 20 \mathrm{FDA}$ ,of\%20COVID $\% 2 \mathrm{D} 19 \% 20$ requiring $\% 20$ hospitalization (Accessed: 10 May 2021).

[8] Plummer E, Buck MD, Sanchez M, Greenbaum JA, Turner J,
Grewal R, et al. Dengue Virus Evolution under a Host-Targeted Antiviral. Journal of Virology. 2015; 89: 5592-5601.

[9] Warfield KL, Schaaf KR, DeWald LE, Spurgers KB, Wang W, Stavale E, et al. Lack of selective resistance of influenza a virus in presence of host-targeted antiviral, UV-4B. Scientific Reports. 2019; 9: 7484

[10] Franco EJ, Pires de Mello CP, Brown AN. Antiviral Evaluation of UV-4B and Interferon-Alpha Combination Regimens against Dengue Virus. Viruses. 2021; 13: 771.

[11] Liu J, Li Y, Liu Q, Yao Q, Wang X, Zhang H, et al. SARS-CoV-2 cell tropism and multiorgan infection. Cell Discovery. 2021; 7: 17.

[12] Yang L, Tu L. Implications of gastrointestinal manifestations of COVID-19. The Lancet Gastroenterology \& Hepatology. 2020 5: 629-630.

[13] Xiao F, Tang M, Zheng X, Liu Y, Li X, Shan H. Evidence for Gastrointestinal Infection of SARS-CoV-2. Gastroenterology. 2020; 158: 1831-1833.e3.

[14] Mossel EC, Huang C, Narayanan K, Makino S, Tesh RB, Peters CJ. Exogenous ACE2 expression allows refractory cell lines to support severe acute respiratory syndrome coronavirus replication. Journal of Virology. 2005; 79: 3846-3850.

[15] Brown AN, Strobel G, Hanrahan KC, Sears J. Antiviral Activity of the Propylamylatin ${ }^{T M}$ Formula against the Novel Coronavirus SARS-CoV-2 In Vitro Using Direct Injection and Gas Assays in Virus Suspensions. Viruses. 2021; 13: 415.

[16] National Library of Medicine. Safety and Pharmacokinetics of UV-4B Solution Administered Orally as Multiple Ascending Doses to Healthy Subjects. 2016. Available at: https://Clinical Trials.gov/show/NCT02696291 (Accessed: 9 May 2021).

[17] Clarke EC, Nofchissey RA, Ye C, Bradfute SB. The iminosugars celgosivir, castanospermine and UV-4 inhibit SARS-CoV-2 replication. Glycobiology. 2021; 31: 378-384.

[18] Reyes H, Du Y, Zhou T, Xie X, Shi PY, Weiss S, et al. Glucosidase inhibitors suppress SARS-CoV-2 in tissue culture and may potentiate. BioRxiv. 2021. (in press)

[19] Zhao X, Guo F, Comunale MA, Mehta A, Sehgal M, Jain P, et al. Inhibition of endoplasmic reticulum-resident glucosidases impairs severe acute respiratory syndrome coronavirus and human coronavirus NL63 spike protein-mediated entry by altering the glycan processing of angiotensin i-converting enzyme 2 . Antimicrobial Agents and Chemotherapy. 2015; 59: 206-216.

[20] Fernández A. Glycosylation of SARS-CoV-2 Steers Evolutionary Outcomes in the Postvaccination Phase. ACS Pharmacology \& Translational Science. 2021; 4: 410-412.

[21] Fukushi M, Yoshinaka Y, Matsuoka Y, Hatakeyama S, Ishizaka $\mathrm{Y}$, Kirikae T, et al. Monitoring of S protein maturation in the endoplasmic reticulum by calnexin is important for the infectivity of severe acute respiratory syndrome coronavirus. Journal of Virology. 2012; 86: 11745-11753.

[22] Zhang L, Jackson CB, Mou H, Ojha A, Peng H, Quinlan BD, et al. SARS-CoV-2 spike-protein D614G mutation increases virion spike density and infectivity. Nature Communications. 2020; 11: 6013.

[23] Brufsky A, Lotze MT. DC/L-SIGNs of hope in the COVID-19 pandemic. Journal of Medical Virology. 2020; 92: 1396-1398.

[24] Huang SW, Wang SF. SARS-CoV-2 Entry Related Viral and Host Genetic Variations: Implications on COVID-19 Severity, Immune Escape, and Infectivity. International Journal of Molecular Sciences. 2021; 22: 3060 